\title{
Wirtschaftsunterricht aus der Sicht von Lehrpersonen
}




\section{Vera Kirchner}

\section{Wirtschaftsunterricht \\ aus der Sicht von \\ Lehrpersonen}

Eine qualitative Studie zu fachdidaktischen teachers' beliefs in der ökonomischen Bildung

Springer VS 
Vera Kirchner

Oldenburg, Deutschland

Zugleich Dissertation an der Carl von Ossietzky Universität Oldenburg im Jahr 2015

ISBN 978-3-658-10831-1

ISBN 978-3-658-10832-8 (eBook)

DOI 10.1007/978-3-658-10832-8

Die Deutsche Nationalbibliothek verzeichnet diese Publikation in der Deutschen Nationalbibliografie; detaillierte bibliografische Daten sind im Internet über http://dnb.d-nb.de abrufbar.

Springer VS

(C) Springer Fachmedien Wiesbaden 2016

Das Werk einschließlich aller seiner Teile ist urheberrechtlich geschützt. Jede Verwertung, die nicht ausdrücklich vom Urheberrechtsgesetz zugelassen ist, bedarf der vorherigen Zustimmung des Verlags. Das gilt insbesondere für Vervielfältigungen, Bearbeitungen, Übersetzungen, Mikroverfilmungen und die Einspeicherung und Verarbeitung in elektronischen Systemen.

Die Wiedergabe von Gebrauchsnamen, Handelsnamen, Warenbezeichnungen usw. in diesem Werk berechtigt auch ohne besondere Kennzeichnung nicht zu der Annahme, dass solche Namen im Sinne der Warenzeichen- und Markenschutz-Gesetzgebung als frei zu betrachten wären und daher von jedermann benutzt werden dürften.

Der Verlag, die Autoren und die Herausgeber gehen davon aus, dass die Angaben und Informationen in diesem Werk zum Zeitpunkt der Veröffentlichung vollständig und korrekt sind. Weder der Verlag noch die Autoren oder die Herausgeber übernehmen, ausdrücklich oder implizit, Gewähr für den Inhalt des Werkes, etwaige Fehler oder Äußerungen.

Gedruckt auf säurefreiem und chlorfrei gebleichtem Papier

Springer Fachmedien Wiesbaden ist Teil der Fachverlagsgruppe Springer Science+Business Media (www.springer.com) 


\section{Danksagung}

Das vorliegende Buch befasst sich mit Vorstellungen von Wirtschaftslehrpersonen zum Wirtschaftsunterricht und ist das Ergebnis eines intensiven, nicht nur fachdidaktischen Forschungs- und Entwicklungsprozesses - meiner Promotion am Institut für ökonomische Bildung an der Carl von Ossietzky Universität Oldenburg. Zahlreichen Menschen bin ich für die fortwährende Unterstützung auf diesem Weg sehr dankbar:

$\mathrm{Zu}$ allererst meinem Doktorvater und Mentor Prof. Dr. Dirk Loerwald, der mich in Kiel zunächst für Umweltökonomik und dann für die ökonomische Bildung begeisterte, der mir die Chance eröffnete, in Oldenburg zu promovieren und der mich in den letzten Jahren stets und in sehr hohem Maße gefördert und unterstützt - aber auch herausgefordert hat. Seine Begeisterung für die ökonomische Bildung und sein fachdidaktisches Verständnis haben mich stark geprägt und ich bin ihm für all das, was ich in den letzten Jahren von ihm lernen konnte, sehr dankbar. Er hat mich immer bestärkt, Herausforderungen anzunehmen und mir mit seinem Vertrauen oft den nötigen Rückenwind gegeben.

Dank gilt außerdem Prof. Dr. Dr. h. c. Hans Kaminski für den Vertrauensvorschuss in Form eines Promotionsstipendiums im ersten Jahr sowie den vielen Kolleginnen und Kollegen am Institut für Ökonomische Bildung, die mich auf die eine oder andere Weise bei der Promotion unterstützt haben. Stephan Friebel gilt besonderer Dank für die Intercodierung, Arne Stemmann für den Zusammenhalt und die vielen gemeinsamen Mittagspausen.

Vor allem gilt der Dank aber auch den Wirtschafts- und PolitikWirtschafts-Lehrpersonen, die mir Einblick in ihre Vorstellungen gewährt haben. Ohne ihre Bereitschaft diese Interviews zu führen, wäre das Forschungsvorhaben nicht zu realisieren gewesen.

Prof. Dr. Franziska Birke hat mich durch ihr Forschungsprojekt zu Schülervorstellungen und ihre Überlegungen $\mathrm{zu}$ conceptual change inspiriert, mich mit Lehrervorstellungen $\mathrm{zu}$ beschäftigen und die Dissertation dankenswerterweise als Zweitgutachterin unterstützend begleitet.

Bei der Deutschen Gesellschaft für Ökonomische Bildung (DeGÖB) habe ich früh in der Entstehung dieses Projekts nicht nur, aber 
wesentlich auch im Rahmen des Nachwuchsforums Raum für (fachdidaktischen) Austausch gefunden, für den ich sehr dankbar bin. Auch hier sind aus Kolleginnen und Kollegen vielfach Freunde geworden.

An verschiedenen Stellen hat diese Dissertation in den letzten Jahren hohe Opportunitätskosten gefordert. Dafür, dass sie dieses Vorhaben und mich stets (mit-)getragen und auf verschiedene Weise unterstützt haben, bin ich meiner Familie und meinen Freundinnen und Freunden sehr dankbar. Großer Dank gilt besonders meiner Promotionsfreundin Andrea Albers, Kaja Sörensen, Christina Schnell und Corinna Kardel - nicht nur, aber auch ganz wesentlich für das Feedback zu dieser Arbeit.

Tania, Susanne und Prof. Dr. Holger Kirchner bin ich von Herzen und in besonderer Weise für ihre Unterstützung und ihre Begleitung auf meinem Weg (zum Doktortitel) dankbar.

Vera Kirchner 


\section{Inhaltsverzeichnis}

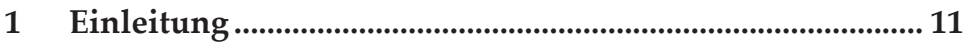

2 Ökonomische Bildung und ihre Rahmenbedingungen .... 15

2.1 Fachdidaktische Elemente ökonomischer Bildung......................... 15

2.1.1 Teil einer zeitgemäßen Allgemeinbildung .................................... 16

2.1.2 Qualifizierung für ökonomische Lebenssituationen....................19

2.1.3 Mündigkeit als übergeordnete Leitidee ......................................... 24

2.1.4 Ökonomischer Denkansatz als Heuristik ..................................... 27

2.1.5 Erwerb ökonomischer Kompetenz(en) .......................................... 30

2.1.6 Auswahl und Rekonstruktion ökonomischer Inhalte................... 34

2.1.7 Handlungsorientierung als konstitutives Element ......................39

2.1.8 Bedeutung fachspezifischer Makromethoden .............................. 41

2.2 Rahmenbedingungen ökonomischer Bildung.................................. 46

3 Vorstellungen (beliefs) ................................................................ 55

3.1 Terminologie und begriffliche Klärung ........................................... 57

3.2 Formale Merkmale und Systematisierung in Forschungsfelder .. 61

3.3 Eigenschaften und Funktionen von Vorstellungen ........................... 65

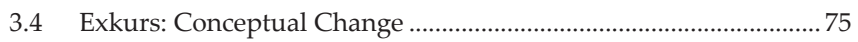

3.5 Vorstellungsdefinition und ihre Implikationen. ............................... 78

3.6 Abgrenzung: Wissen und Vorstellung ……....................................... 79

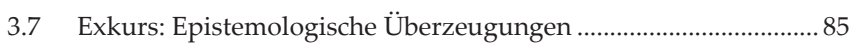

$4 \quad$ Lehrervorstellungen (teachers` beliefs) ................................... 97

4.1 Definition und Eigenschaften von Lehrervorstellungen ............... 98

4.2 Lehrervorstellungen als Dimension professioneller Kompetenz102

4.3 Lehr-lern-theoretische Bedeutung von Lehrervorstellungen ..... 109

4.4 Lehrervorstellungen und Unterrichtshandeln ............................... 112

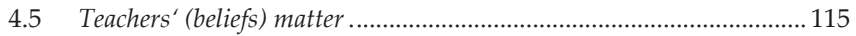

4.6 Status quo und Studien in der Lehrervorstellungsforschung..... 120

4.7 Lehrervorstellungsforschung in den Sozialwissenschaften ......... 127

4.8 Systematisierung von Lehrervorstellungen..................................... 133 
4.9 Epistemologische Überzeugungen von Lehrpersonen 142

4.10 Ansätze und Methoden 147

5 Methodische Grundlagen und Forschungsdesign 153

5.1 Prämissen und grundlegende Überlegungen. 154

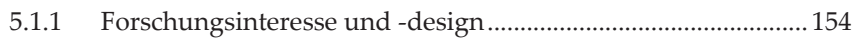

5.1.2 Der Umgang mit Vorwissen und Offenheit ................................. 162

5.1.3 Qualitative Gütekriterien und ihre Umsetzung.......................... 165

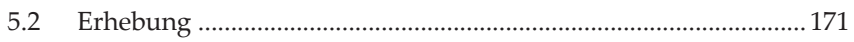

5.2.1 Problemzentriertes Interview als Erhebungsmethode .............. 171

5.2.2 Qualitativer Stichprobenplan und Analyse des Samples......... 174

5.2.3 Design und Erläuterung der Erhebungsinstrumente................ 185

5.3 Auswertung: Qualitative Inhaltsanalyse......................................... 192

5.3.1 Reduzierende qualitative Inhaltsanalyse ................................... 192

5.3.2 Ablauf induktive Kategorienbildung .......................................... 195

5.3.3 Intercodierungsprozess: Vorgehensweise und Ergebnisse...... 200

6 Vorstellungen der Wirtschaftslehrpersonen....................... 205

6.1 Vorstellungen zum Wirtschaftsunterricht .................................... 207

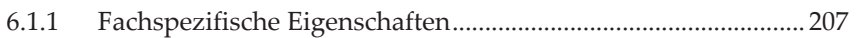

6.1.2 Abgrenzung zu anderen Schulfächern......................................... 220

6.2 Vorstellungen zum Lernen und Lehren ......................................... 225

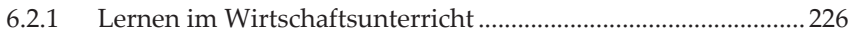

6.2.2 Lehren im Wirtschaftsunterricht ................................................. 243

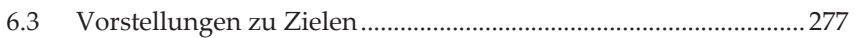

6.3.1 Fachliche Ziele des Wirtschaftsunterrichts. ................................. 280

6.3.2 Beitrag zu fächerübergreifenden Zielen ..................................... 288

6.4 Vorstellungen zu Inhalten ................................................................ 291

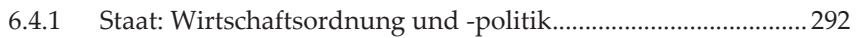

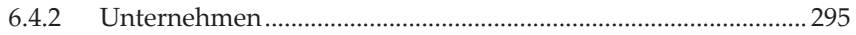

6.4.3 Private Haushalte........................................................................... 297

6.4.4 Ökonomische Prinzipien, Theorien und Modelle ...................... 299

6.4.5 Berufsorientierung ..................................................................... 303

6.4.6 Internationale Wirtschaftsbeziehungen ........................................ 304

6.4.7 Ökonomie und Ökologie sowie Wirtschaft vor Ort .................... 306

6.5 Vorstellungen zu Methoden des Wirtschaftsunterrichts.............. 307 
6.5.1 Makro-, Mikromethoden und Sozialformen ................................. 307

6.5.2 Methodeneinsatz und -umsetzung ............................................... 315

6.6 Vorstellungen zur Wirtschaft im Wirtschaftsunterricht............... 319

6.6.1 Grundsätzliche Befürwortung und Kritik an der Umsetzung 319

6.6.2 Gestaltung von Praxiskontakten ................................................. 325

6.7 Vorstellungen zu den Rahmenbedingungen................................. 330

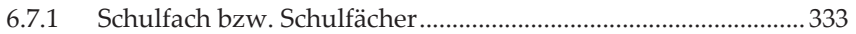

6.7.2 Lehr-Lern-Materialien und Ausstattung........................................ 340

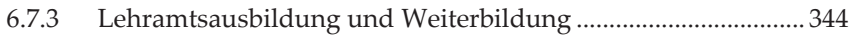

6.7.4 Curricula, Richtlinien und andere Vorgaben................................ 348

7 Zusammenfassung - Reflexion - Implikationen ................ 351

7.1 Lehrervorstellungen zum Wirtschaftsunterricht ........................... 352

7.2 Übereinstimmungen und Unterschiede zur Fachdidaktik ..........362

7.3 Einordnung und fachspezifische Besonderheiten .......................... 369

7.4 Fachdidaktische Implikationen der vorliegenden Arbeit............. 371

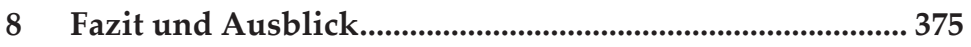

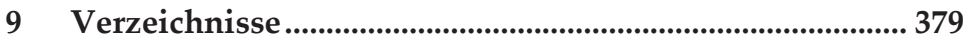

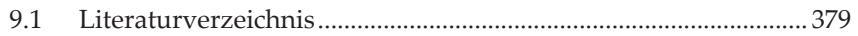

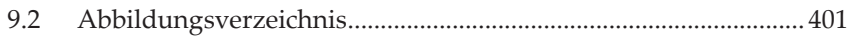

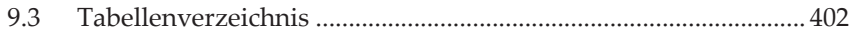

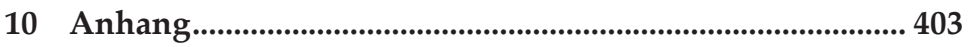

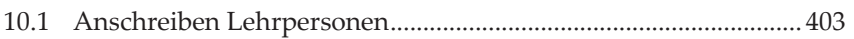

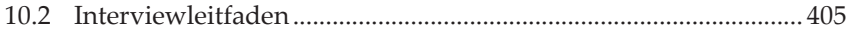

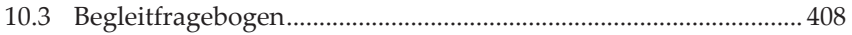

10.4 Selektionskriterien und ihre Definitionen........................................ 409 